mit gewöhnlichen statistischen Methoden nur dann erwarten, daß nicht unsinnige Wahrscheinlichkeitswerte herauskommen, wenn wir den Ursprung so legen, daß alle Prozesse, die von ihm zu irgendeinem andern Punkt führen, immer in dieselbe Richtung, hier speziell in die Richtung abnehmender $|w|$ weisen. Wir müssen also stets

$$
|w| \leq w_{\max } \leq|\stackrel{\circ}{w}|
$$

annehmen. Darin ist $w_{\max }$ das Maximum der Werte von $|w|$, das in einer konkreten Gesamtheit wirklich erreicht wird.

Setzen wir $|\stackrel{\circ}{w}|=1$, so ist der Ursprung ein Punkt der Gewißheit. Wir gelangen zu einer Darstellung, in der die Gesamtheit als reiner Fall erscheint. Setzen wir $|\stackrel{\circ}{w}|=w_{\max }$, so erhalten wir, wenn $w_{\max }<1$ ist, ein Gemisch. Dasselbe gilt für irgendwelche Zwischenwerte:

$$
w_{\max } \leq|\stackrel{\circ}{w}|<1 .
$$

Eine andere Wahl für positive $W_{i k}$ und $v_{i}$ gibt es nicht. Wenn $w_{\max }=1$ ist, bleibt nur der reine Fall übrig.

Wir formulieren zum Schluß das Ergebnis: Der Grundprozeß der Quantentheorie enthält keine Elemente, die nicht der Anschauung zugänglich wären oder die sich der Objektivierbarkeit entzögen. Als Beweis dafür betrachten wir den Umstand, daß es stets möglich ist, eine statistische Gesamtheit von Grundprozessen mit den gewöhnlichen Methoden der Statistik zu behandeln, wenn man dabei nur berücksichtigt, daß diese für gerichtete Prozesse geschaffen sind, während die Quantenstatistik rückläufig sein kann. Hierin ist es begründet, daß wir den zeitlichen Verlauf von einem gemeinsamen Ursprung her betrachtet haben und nicht differentiell, wie es die Kausalanalyse erfordert. Die umkehrbaren statistischen Vorgängen angemessene Methode ist mindestens bei zweiwertigenWahrscheinlichkeiten (Alternativen) die der Quantenstatistik.

\title{
Über die Objektivierbarkeit der Zustände in der nichtrelativistischen Quantenmechanik
}

\author{
Von Rudolf HaAg
}

Aus dem Institut für theoretische Physik der Universität München

(Z. Naturforschg. 8a, 13-16 [1953]; eingegangen am 2. Oktober 1952)

\begin{abstract}
Erwin Fues zum 60. Geburtstag gewidmet in dankbarer Erinnerung an viele Gespräche
\end{abstract}
\begin{abstract}
Auf Grund zweier allgemeiner Forderungen an den Zustandsbegriff wird die Möglichkeit diskutiert, einem quantenmechanischen Einzelsystem objektive Zustandsgrößen zuzuordnen. Als kleinste hierdurch zugelassene Zustandsmannigfaltigkeit ergibt sich die Gesamtheit aller Wellenfunktionen. Da andererseits eine reale Auffassung der Wellenfunktionen zu den oft diskutierten Schwierigkeiten führt, erscheint die Anwendung des Zustandsbegriffs im hier definierten Sinn bei quantenmechanischen Systemen nicht zulässig.
\end{abstract}

Z wei Aspekte der Quantenmechanik sind verantwortlich für die Revision der erkenntnistheoretischen Grundlagen der Physik, die mit der Entwicklung der Quantenmechanik einsetzte und in der Formulierung der Axiome der Transformationstheorie einen gewissen Abschluß fand:

1. Die Indeterminiertheit des elementaren Geschehens. D.h.: Die bestmögliche Kenntnis der Situation der gegenwärtigen Welt genügt im allg. nicht, um den Ausgang eines Experiments vorherzusagen.
2. Die Schwierigkeiten der Objektivierbarkeit der Eigenschaften eines Systems. D. h.: Es ist im allg. nicht sinnvoll, einer Meßgröße in einem System einen bestimmten Wert zuzuschreiben, solange sie nicht beobachtet wird.

Hier soll lediglich die Schwierigkeit 2 . betrachtet werden. Es besteht gedanklich kein zwingender Grund, den Begriff „Zustand eines Einzelsystems“" unmittelbar an die Meßgrößen anzuschließen. Man kann auch zunächst die Forderungen fixieren, die man an den Begriff stellt, und dann fragen, ob es 
irgendwelche Größen gibt, die diese Forderungen erfüllen und aus deren Gesetzmäßigkeiten sich die Erfahrungsgesetze - die auf Systemgesamtheiten bezogen sind - ableiten. In diesem weiteren Sinn möge das Wort „Objektivierbarkeit" hier verstanden werden. Es ist klar, daß die in 2 . gegebene Fassung dieses Wortes sich unterordnet und da $\beta$ außerdem ein System mit determinierten Gesetzen trivialerweise objektive Zustände besitzt. Umgekehrt folgt aus 1 . und 2 . noch nicht ohne weiteres die Nichtobjektivierbarkeit im weiteren Sinn. Da schon die Behauptung 2. und erst recht die allgemeinere Fragestellung keine direkte Aussage über den empirischen Tatbestand, sondern erst eine solche über die Möglichkeiten seiner Deutung betrifft, liegt zunächst der Verdacht nahe, die Diskussion sei inhaltsleer. Indessen ist eine direkte Prüfung der Frage, ob etwa eine Größe einen Wert hat, solange man ihn nicht mißt, natürlich nicht erst in der Quantenphysik unmöglich. Man kann daher die Frage nur so verstehen: führt ihre Bejahung zuWidersprüchen und Komplikationen oder zu einer Vereinfachung der Begriffsbildung? Wir fragen also: Welche Konsequenzen hätte die Annahme objektiver Größen in der Quantenphysik?

\section{Objektivierbarkeit der Meßgrößen}

Es möge zunächst nochmals die engere Aussage 2 . betrachtet werden, da hier die Diskussion ohne formale Hilfsmittel zu führen ist. Heisenberg ${ }^{1}$ diskutiert das folgende Beispiel: In einem stationären Zustand eines Elektrons im Atomverband der Energie $E$ besteht eine beträchtliche Wahrscheinlichkeit dafür, daß seine kinetische Energie negativ sein müßte, wenn die Beziehung

$$
E=E_{\text {kin }}+E_{\text {pot }}(\mathfrak{r})
$$

gelten würde und der Ort einen objektiven Sinn hätte. Ein negatives $E_{\text {kin }}$ wird aber ausgeschlossen, wenn es eine objektive Bedeutung (d. h. eine Bedeutung unabhängig vom Meßakt) haben soll, da niemals negative kinetische Energien gemessen wurden. Es gibt nur eine Alternative zur Nichtobjektivierbarkeit: Die Relation (1) gilt in der Quantenmechanik nicht. Da sie aber für die Mittelwerte innerhalb beliebiger Gesamtheiten richtig ist, würde folgen, daß an die Stelle der klassischen funktionalen Beziehung der Größen eine statistische Korre-

\footnotetext{
${ }^{1} \mathrm{~W}$. Heisen berg, Die Physikalischen Prinzipien der Quantentheorie. Leipzig 1944, S. 25.
}

lation treten müßte. In ähnlicher Weise kann man bei anderen Meßgrößen schließen. Das Ergebnis wäre: die Zahl der Freiheitsgrade müßte ungeheuer groß sein und vermutlich alle Meßgrößen umfassen.

\section{Objektivierbarkeit allgemeiner Zustandsgrößen}

Wir stellen für das folgende zwei Forderungen an den Begriff Zustand:

a) Jedes System einer Gesamtheit befindet sich zu einem bestimmten Zeitpunkt in einem und nur einem Zustand, der durch dieWerte irgendwelcher Variabler, die wir kollektiv mit $\xi$ bezeichnen, charakterisiert wird.

b) Macht man an einem System im Zustand $\xi$ irgendein Experiment, so soll der Ausgang (ob statistisch oder determiniert) durch $\xi$ allein bestimmt sein (Vollständigkeitsforderung).

Mit diesen beiden Forderungen ist eine Fülle von Möglichkeiten umfaßt, über die wir hier keine näheren Voraussetzungen machen wollen. Ob etwa die $\xi$-Größen in irgendeinerWeise direkt den Meßgrößen $Q$ des Systems zugeordnet werden können oder ob letztere erst bei dem hier nicht analysierten Wechselwirkungsakt mit dem Beobachtungsapparat, ,entstehen" und im unbeobachteten System keine Bedeutung haben, ob also der UUbergang von einem Zustand $\xi_{\alpha}$ zu einem Meßergebnis $q_{\kappa}$ determiniert ist oder nicht, soll nicht betrachtet werden ${ }^{2}$. Ebensowenig fragen wir, ob die zeitliche Veränderung der Zustände $\xi$ selbst in determinierter Weise vor sich geht, ob die Herstellung einer Gesamtheit von Systemen in einem wohldefinierten Zustand experimentell möglich ist. Ja sogar die Frage, ob neben $\xi$ noch irgendwelche verborgenen Variablen $\eta$ existieren könnten, ist für das folgende belanglos, solange die Existenz von $\eta$ nicht der Forderung b) widerspricht, d. h. sich in der Statistik der quantenmechanischen Versuche äußert. Wenn dies der Fall wäre, so wären die $\eta$ eben keine verborgenen Parameter, sondern müßten per definitionem in die Bestimmungsstücke $\xi$ aufgenommen werden. Dagegen wäre die Existenz von $\eta$ mit b) verträglich, wenn etwa bei allen Gesamtheiten, die in der Quantenmechanik untersucht werden, jedem $\xi$ automatisch eine bestimmte Häufigkeitsverteilung über $\eta \mathrm{zu}$ käme.

${ }^{2}$ Die Indizes $\alpha$ bzw. $k$ sollen einen speziellen Zustand bzw. Meßwert charakterisieren. 
Man kann nun folgendermaßen schließen: Jede statistische Gesamtheit von Systemen muß nach a) dargestellt werden durch eine Verteilungsfunktion $W(\xi)$, jedes Experiment nach b) durch eine Matrix $S_{q_{k}{ }_{\alpha}}$, die die Wahrscheinlichkeit des Meßwerts $q_{k}$ beim Vorliegen des Zustandes $\xi_{\alpha}$ darstellt. Dabei muß $S$ nach b) unabhängig sein von $W(\xi)$ und da der Versuch an jedem System der Gesamtheit unabhängig von den anderen durchgeführt wird muß für die Wahrscheinlichkeit des Meßwerts $q_{k}$ innerhalb der Gesamtheit gelten.

$$
W\left(q_{k}\right)=\sum_{\alpha} S_{q_{k} \xi_{\alpha}} W\left(\xi_{\alpha}\right) .
$$

Also ein linearer Zusammenhang zwischen den Wahrscheinlichkeiten. Vergleichen wir damit die Aussagen der nichtrelativistischen Quantenmechanik! Eine beliebige Gesamtheit wird dargestellt durch die statistische Matrix $U$ und dieWahrscheinlichkeit für $q_{k}$ ist

$$
W\left(q_{k}\right)=\operatorname{Spur}\left(P_{q_{k}} U\right),
$$

wenn $P_{q_{k}}$ der zum Meßwert $q_{k}$ gehörende Projektionsoperator ist. Also ein linearer Zusammenhang zwischen $W\left(q_{k}\right)$ und $U$. Wenn die Aussagen (2) und (3) äquivalent sein sollen, so muß jedem $W\left(\xi_{\alpha}\right)$ ein $U$ zugeordnet werden können und entsprechend jedem $S_{q_{k} \Sigma_{\alpha}}$ ein $P_{q_{k}}$. Der Vergleich von (2) und (3) zeigt weiter, daß der Zusammenhang zwischen $W(\xi)$ und $U$ einerseits, $P_{q_{k}}$ und $S$ andererseits ein linearer sein muß. Also

$$
W\left(\xi_{\alpha}\right)=\sum_{r s} T_{s r}^{\alpha} U_{r s}=\operatorname{Spur}\left(T^{\alpha} U\right) .
$$

Es folgt aus (2) und (3):

$$
W\left(q_{k}\right)=\operatorname{Sp} \underset{\alpha}{\sum} S_{q_{k} \xi_{\alpha}} T^{\alpha} U=\operatorname{Sp} P_{q_{k}} U
$$

für beliebige $U$, also

$$
P_{q_{k}}=\sum_{\alpha} S_{q_{k} \lesssim \alpha} T^{\alpha}
$$

d. h. es soll ein beliebiger Projektionsoperator zerlegt werden nach einem fest gegebenen System von Operatoren $T^{\alpha}$, die hermitesch und positiv definit angenommen werden müssen, damit bei beliebigem $U$ ein positives $W\left(\xi_{\alpha}\right)$ resultiert. Die Koeffizienten $S_{q_{k}{ }_{\alpha}}$ sollen dabei positive, reelle Größen sein, denn sie bedeuten. Übergangswahrscheinlichkeiten.

${ }^{3}$ Für den Fall des $n$-Punkt-Raums hat die hermitesche Matrix $U n^{2}$ reelle Bestimmungsstücke, denen die $n^{2}$ Wertekombinationen der klassischen Zustandsgrößen $q_{i}, p_{K}(i, k=1 \ldots n)$ gegenüberstehen.
Man kann die Frage auch so formulieren: Was ist das kleinste System positiv definiter Matrizen $T^{\alpha}$, aus denen man durch Linearkombination mit positiven Koeffizienten jede beliebige positiv definite Matrix aufbauen kann? Die Antwort lautet: Die Gesamtheit aller Projektionsoperatoren. Denn ein Projektionsoperator ist nicht als Linearkombination zweier Matrizen im obigen Sinn darzustellen.

Beweis : Angenommen wir hätten eine solche Zerlegung:

$$
P_{\varphi}=\alpha U_{1}+(1-\alpha) U_{2} .
$$

Ist $(\varphi \psi)=0$, dann hat man für die Erwartungswerte hinsichtlich $\psi$ :

$$
0=\alpha<\left|U_{1}\right|>+(1-\alpha)<\left|U_{2}\right|>.
$$

Da $\alpha,(1-\alpha),<\left|U_{1}\right|>$ und $<\left|U_{2}\right|>$ nicht negativ sein dürfen, ist nur eine triviale Lösung möglich.

Damit ist gezeigt: Die kleinste Mannigfaltigkeit der objektiven Zustände eines Systems, die mit der Vollständigkeitsforderung im Einklang ist, ist die Gesamtheit aller Wellenfunktionen.

Es sei hier erwähnt, daß Gln. (2), (3), (4) mit einer sehr viel kleineren Zustandsmannigfaltigkeit zu erfüllen sind, wenn man erlaubt, daß die Übergangswahrscheinlichkeiten $S$ negative Werte annehmen können, was aber natürlich im Rahmen dieser Überlegung nicht in Betracht gezogen wird. Die kleinste Mannigfaltigkeit ist in diesem Fall gleich der der Matrixelemente von $U$ und stimmt überein mit der korrespondierenden klassischen Zustandsmannigfaltigkeit ${ }^{3}$. Man kann daher etwa $\xi$ mit den Wertepaaren zweier nicht gleichzeitig meßbarer Größen $Q$ und $P$ identifizieren und erhält dann

$$
W\left(q_{k}, p_{r}\right)=1 / 2\left(T_{k r} U_{k r}+T_{k r}^{*} U_{k r}^{*}\right) .
$$

(Keine Summation!)

Dabei ist $U_{k r}$ das Matrixelement von $U$ in der gemischten Darstellung, welche die Zeilen nach den Eigenwerten von $Q$, die Spalten nach denen von $P$ ordnet, und $T_{k r}$ das Element der unitären Transformationsmatrix, welche die $Q$-Darstellung in die $P$-Darstellung überführt.

Gl. $\left(4^{\prime}\right)$ wird $u$. a. in den Untersuchungen von Bopp über den Zusammenhang zwischen Korrelationsstatistik und Quantentheorie diskutiert ${ }^{4}$.

${ }^{4}$ F. Bopp, Z. Naturforschg. 7a, 82 [1952]; 2a, 202 [1947]. 


\section{Konsequenzen}

I. Es gibt (im wesentlichen) eine einzige Möglichkeit, die Vorstellung objektiver Zustände des Einzelsystems im Sinn der Forderungen a) und b) in der Quantenmechanik aufrechtzuerhalten ${ }^{5}$. Diese Möglichkeit: die Auffassung von $\psi$ als objektivem Ausdruck für den Systemzustand, ist bereits häufig diskutiert worden. Sie führt $\mathrm{zu}$ Schwierigkeiten, wenn man die Wechselwirkung zweier Systeme in Betracht zieht ${ }^{6}$ und hat außerdem die merkwürdige Konsequenz, daß jede Gesamtheit äquivalent ist einer speziellen Gesamtheit, in der nur ein Teil der Zustände (die Wellenfunktionen eines einzigen Orthogonalsystems) vorkommen.

II. Die Argumentation in 2. hat Berührungs-

5 Man kann sich natürlich beliebig viele umfassendere Zustandsmannigfaltigkeiten ausdenken, doch besteht dazu im Rahmen unserer Forderungen kein Anla $\$$ und es wird nichts dadurch gewonnen. punkte mit dem Beweis, den v. Neumann ${ }^{7}$ für die Indeterminierbarkeit der Quantenmechanik gegeben hat. In der Tat ist die Aufgabe (5), auf die das Problem reduziert wurde, identisch mit der Frage, nach den ,reinen" Gesamtheiten bei v. Neumann. Man kann also sagen: die Forderungen a) und b) an den Zustandsbegriff verlangen zumindest, daß eine Gesamtheit von Systemen im gleichen Zustand $\xi$ eine ,,reine" Gesamtheit sei. Im Gegensatz zu dem erwähnten Indeterminierbarkeitsbeweis kommt es bei der Frage nach der kleinsten Zustandsmannigfaltigkeit jedoch nicht darauf an, daß keine Gesamtheiten existieren, die nicht durch eine statistische Matrix $U$ beschrieben werden können, sondern nur darauf, daß jedes $U$ durch eine Gesamtheit realisierbar ist.

${ }^{6}$ W. Heisenberg, S. Zitat 1: Schnittbeweis; Einstein, Podolsky u. Rosen, Physic. Rev. 47, 777 [1935].

7 J. v. Neumann: Die mathematischen Grundlagen der Quantenmechanik, Springer Verlag.

\title{
Zur Theorie der Streuung von Elektronen an Elektronen
}

\author{
Von H. SALECKER
}

Aus dem Institut für theoretische und angewandte Physik der Technischen Hochschule Stuttgart

(Z. Naturforschg. 8a, 16-19 [1953]; eingegangen am 18. November 1952)

Herrn Professor Dr. Erwin Fues zum 60. Geburtstag

\begin{abstract}
Im Hinblick auf eine experimentelle Prüfung der höheren Näherungen der Quantenelektrodynamik mit Hilfe der Streuung von Elektronen an Elektronen werden die Abänderungen der Elektron-Elektron-Streuung untersucht, die sich aus der verallgemeinerten Feldtheorie ergeben. Im ersten Abschnitt wird das allgemeine Matrixelement für eine beliebige Strukturfunktion angegeben. Im zweiten Abschnitt wird der differentielle Wirkungsquerschnitt für eine Spezialisierung berechnet und der allgemeine Wirkungsquerschnitt mit Hilfe des berechneten dargestellt. Zum Schluß werden die Abweichungen von der Møllerschen Formel diskutiert, die sich bei zwei verschieden gewählten Größen der Abänderung (des ,Elektronenradius“") ergeben. Dabei zeigt es sich, daß die betrachteten Abweichungen bei einer plausiblen Größe der Abänderung erst dann deutlich bemerkt werden können, wenn die Energie des einfallenden Teilchens im Laboratoriumssystem $10^{9} \mathrm{eV}$ überschreitet.
\end{abstract}

$\mathrm{D}^{\mathrm{i}}$ ie höheren Näherungen der Quantenelektrodynamik sind mit groler Genauigkeit durch zwei Experimente bestätigt. Das sind bekanntlich die Messungen des zusätzlichen magnetischen Moments des Elektrons und der Lambschen Verschiebung bei der Feinstruktur des Wasserstoffs. Diese beiden Effekte hängen jedoch von den Einzelheiten der Theorie wesentlich nur im nichtrelativistischen Gebiet, d. h. vom Bereich kleiner Energie-ImpulsÜbertragung ab. Es sind deshalb quantitative Prü- fungen der höheren Näherungen der Quantenelektrodynamik im Bereich großer Energie-Impuls-Übertragungen nötig. Dazu kommen besonders Streuexperimente in Frage. Die Streuung von Elektronen an Kernen, an die man dabei zunächst denkt, ist jedoch hierzu nicht sehr geeignet, da die Ladungsverteilung der Kerne nicht genau bekannt ist. Man benutzt vielmehr umgekehrt Abweichungen von der normalen Streuung zur Bestimmung der Ladungsverteilung. Im Rahmen der Quantenelektrodynamik bietet sich 